\title{
Tailoring Molecular Imprinted Polymers as Biomimetic Recognition Coatings for Anti-Diabetic Drugs
}

\author{
Adnan Mujahid $^{1}$, Isma Haq ${ }^{1}$, Tajamal Hussain ${ }^{1}$, Sadia Z. Bajwa ${ }^{2}$, Adeel Afzal ${ }^{3}$ \\ 1 Institute of Chemistry, University of the Punjab, Quaid-i-Azam Campus Lahore 54590, Pakistan \\ ${ }^{2}$ National Institute of Biotechnology and Genetic Engineering, Jhang Road, Faisalabad, Pakistan \\ ${ }^{3}$ Department of Chemistry, College of Science, University of Hafr Al Batin, P.O. Box 1803, Hafr Al \\ Batin 31991, Saudi Arabia \\ adnanmujahid.chem@pu.edu.pk
}

\begin{abstract}
:
Molecular recognition of pharmaceutical molecules in complex mixtures is a subject of significant interest both for clinical analysis and routine quality control assays in pharma industries. The ever increasing use of anti-diabetic drugs e.g. sitagliptin and metformin also demand their selective extraction and quantitative determination. In present study, we tailored molecular imprinted polymer matrix as highly responsive chemical sensor coatings for molecular recognition of above mentioned anti-diabetic drugs. Molecular imprinted coatings were synthesized as poly(methacrylic acid) crosslinked with ethyleneglycol dimethacrylate following free radical initiated polymerization. The imprinted polymer was processed in gel form and integrated with interdigital capacitors (IDCs) as thin layers by spin coating. The prime advantages of using IDCs are their low fabrication cost, rapid response time and ruggedness. The synthesized imprinted sensor layers have shown appreciable response for target analytes comparing to non-imprinted/control material. Therefore, the developed sensor coatings can be suitably used for specific drug analysis and related bioassay of anti-diabetic drugs.
\end{abstract}

Key words: Molecular imprinting, anti-diabetic drugs, IDCs, chemical sensors, sitagliptin, metformin.

\section{Introduction}

The binary formulations of sitagliptin and metformin have shown suitable potential for treating type 2 diabetes. As there is a growing interest in using these drugs for combination therapy since they provides adequate glycemic control [1]. In this perspective, analytical protocols dealing with selective recognition of these drugs in complex mixtures are essentially required for their quantitative estimation.

Molecular imprinting is a well known method for synthesizing synthetic recognition materials [2] having predefined functionality. Aside from typical applications of imprinted polymers, the prime interest in using them is the flexibility in their processing e.g. in micro/nanoparticles as well as thin layers. This allows using molecular imprinted polymers (MIPs) for separation and sensing purposes, respectively. In view of sitagliptin and metformin presence in complex pharmaceutical formulations and biological samples herein, we developed molecularly imprinted materials as highly responsive sensor coatings combined with interdigital capacitors (IDCs) [3] for anti-diabetic drugs.

\section{Experimental}

We prepared molecular imprinted polymer using methacrylic acid (MAA) as functional monomer along with ethylenegylcol dimethacrylate (EGDMA) as cross linker in dimethylformamide (DMF) as solvent. This mixture was heated for 30 minutes at $65^{\circ} \mathrm{C}$ to form pre-polymer complex. After that, azoisobutyronitrile (AIBN) was introduced to initiate free radical polymerization and heating was continued for another 15 minutes. The resultant imprinted polymer was processed as viscous gel which was diluted accordingly for coating IDCs. The comb shaped interdigital electrode pattern was developed by screen printing. The non-imprinted gel was synthesized under the same conditions except adding temple (sitagliptin/metformin). The synthesized polymer gels were developed on IDCs as thin layers by spin coating at 2000rpm. All the sensor measurements were conducted by connecting coated IDCs with Sourcetronic ST2817B LCR meter. 


\section{Discussion}

Molecular imprinted materials can be tailored according to desired application for instance; they can be processed into sorbent particles as well as sensor coatings. A schematic diagram of synthesizing MIP in gel form has been shown as follows. molecules. Furthermore, it can be seen that after washing the sensor layer with methanol, capacitance values approaches back to base line indicating the complete removal of analyte molecules from the layer. This allows the possibility of reusing the sensor surface for successive measurements.

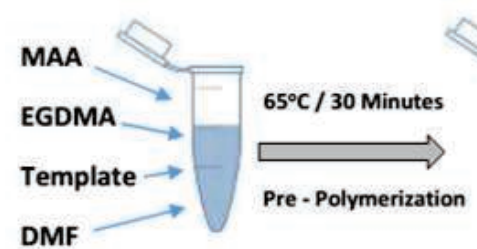

(Template: Sitagliptin / Metformin)

In previous studies [4], we showed that sitagliptin or metformin imprinted particles could be used efficiently for respective drug rebinding. The cross sensitivity of the prepared imprinted particles was substantially high for target drug rebinding. Now, we extended this strategy and processed imprinted polymers as thin layers coated on IDCs. For sensor measurements, the IDCs were connected to LCR meter. On exposing to standard drug concentration, a shift in capacitance was observed which was taken as sensor response. For instance, figure 1 shows the capacitance shifts for sitagliptinimprinted and non-imprinted layers exposed to 10 ppm sitagliptin standard solution.

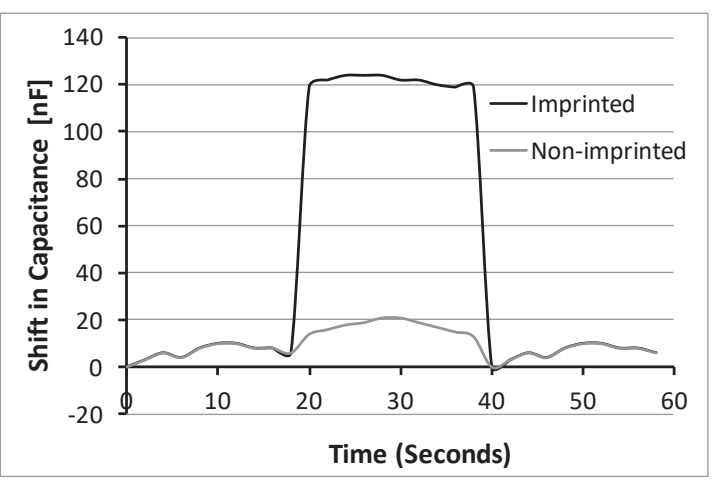

Fig. 1. Sensor response of IDCs coated with sitagliptin-imprinted and non-imprinted layers when exposed to $10 \mathrm{ppm}$ sitagliptin standard solution.

The graph demonstrates that imprinted layer yields much higher sensor shift than nonimprinted material on exposing to sitagliptin solution. This indicates that imprinted layer possesses highly adapted interaction sites for analyte recognition. In imprinted cavities, sitagliptin molecules are accommodated through reversible non-covalent interactions e.g. hydrogen bonding and others. Thus, imprinted sites offer chemical fit to analyte

\section{Conclusion}

Molecular imprinted particles have already shown their potential for extraction of antidiabetic drugs. In this work, we demonstrated that imprinted polymer can be processed as thin films and by combining with IDCs they offer appreciably high sensor response for target pharmaceutical molecules. Additionally, the layer-analyte interactions are reversible which allows reusing sensor interface for several measurements. The use of IDCs as sensing transducer is favorable for obtaining rapid shifts in sensor response and for being low cost they can be fabricated for developing viable sensor devices.

\section{References}

[1] B.J. Goldstein, M.N. Feinglos, J.K. Lunceford, J. Johnson, D.E. Williams-Herman, Effect of Initial Combination Therapy with Sitagliptin, A Dipeptidyl Peptidase-4 Inhibitor, and Metformin on Glycemic Control in Patients with Type 2 Diabetes, Diabetes Care 30, 1979-1987 (2007); doi.org/10.2337/dc07-0627

[2] J. Wackerlig, P.A. Lieberzeit, Molecularly Imprinted Polymer Nanoparticles in Chemical Sensing-Synthesis, Characterisation and application, Sensors and Actuators B: Chemical 207, 144-157 (2015); doi.org/10.1016/j.snb.2014.09.094

[3] A. Mujahid, S. Aigner, F.L. Dickert, Microstructured interdigital capacitors with synthetic antibody receptors for $\mathrm{ABO}$ blood-group typing, Sensors and Actuators B: Chemical 242, 378-383 (2017); doi.org/10.1016/j.snb.2016.11.056

[4] I. Haq, A. Mujahid, A. Afzal, N. Iqbal, S.Z. Bajwa, T. Hussain, K. Shehzad, H. Ashraf, Developing Imprinted Polymer Nanoparticles for the Selective Separation of Antidiabetic Drugs, Journal of Separation Science 38, 3469-3476 (2015); doi; 10.1002/jssc. 201500506 